\title{
AN INERTIAL MANIFOLD AND THE PRINCIPLE OF SPATIAL AVERAGING
}

\author{
HYUKJIN KWEAN
}

(Received 16 January 2001)

\begin{abstract}
We examine the existence of inertial manifold of a class of differential equations with particular boundary conditions.

2000 Mathematics Subject Classification. 34C29, 35P20, 35K57.
\end{abstract}

1. Introduction. In this paper, we examine the existence of an inertial manifold of the scalar-valued reaction-diffusion equation of the form

$$
u_{t}=v \Delta u+f(u), \quad u \in \mathbb{R}
$$

with particular boundary conditions. For (1.1), we consider the following domains $\Omega_{n} \subset \mathbb{R}^{n}, n=2,3$

$$
\begin{aligned}
& \Omega_{2}=\left\{\left(x_{1}, x_{2}\right) \in \mathbb{R}^{2}: 0<x_{2}<\sqrt{3} x_{1}, 0<x_{1}<\frac{\pi}{2}\right\}, \\
& \Omega_{3}=\Omega_{2} \times(0, L \pi),
\end{aligned}
$$

where $L$ is a positive real number satisfying some conditions.

This problem was studied by Mallet-Paret and Sell [2] for 2-dimensional rectangular and 3-dimensional cubic domains and Kwean [1] extended their result into several new domains including the type of domains in (1.2). However for their works, they consider either Dirichlet or Neumann boundary conditions or periodic boundary conditions when the domain is a Cartesian product of intervals.

In our attempt to study (1.1) and (1.2), we consider particular boundary conditions which are different from previous works. In order to formulate boundary conditions we introduce some notations; let $S_{n}$ be the subset of $\partial \Omega_{n}$ in (1.2) given by

$$
\begin{aligned}
& S_{2}=\left\{\left(x_{1}, x_{2}\right) \in \partial \Omega_{2}: x_{1}=\frac{\pi}{2}\right\}, \\
& S_{3}=\left\{\left(x_{1}, x_{2}, x_{3}\right) \in \partial \Omega_{3}: x_{1}=\frac{\pi}{2}\right\},
\end{aligned}
$$

and let $S_{n}^{c}=\partial \Omega_{n} \backslash S_{n}, n=2,3$ and hence $\partial \Omega_{n}=S_{n}^{c} \cup S_{n}$. Then the boundary conditions we study here are given as follows: for each $n=2,3$,

$$
u=0 \quad \text { on } S_{n}^{c}, \quad \frac{\partial u}{\partial n}=0 \quad \text { on } S_{n} .
$$


For this purpose we need good information about the eigenvalues and the eigenfunctions of $-\Delta$ with given boundary conditions (1.4) and then by showing that the differential equations (1.1), (1.2), and (1.4) satisfy all the hypotheses of the Invariant Manifold Theorems (Mallet-Paret and Sell [2]), we obtain the desired result.

2. The eigenvalue problem and the weaker PSA. Now consider the following eigenvalue problems for $n=2,3$ :

$$
\Delta u+\lambda u=0 \quad \text { in } \Omega_{n}, \quad u=0 \quad \text { on } S_{n}^{c}, \quad \frac{\partial u}{\partial n}=0 \quad \text { on } S_{n} .
$$

To solve this problem, first we consider the eigenvalue problem for the following domains:

$$
\tilde{\Omega}_{2}=\{\text { equilateral triangle of side } \pi\}, \quad \tilde{\Omega}_{3}=\tilde{\Omega}_{2} \times(0, L \pi) .
$$

Then we obtain the following result.

LEMmA 2.1. Let $\tilde{\Omega}_{n} \subset \mathbb{R}^{n}$ be given in (2.2) for $n=2,3$. Then the eigenvalues and the eigenfunctions of $-\Delta$ for Dirichlet boundary conditions are of the forms: for $\tilde{\Omega}_{2} \subset \mathbb{R}^{2}$,

$$
\begin{aligned}
\tilde{\lambda}_{k} & =\frac{16}{27}\left(k_{1}^{2}+k_{2}^{2}-k_{1} k_{2}\right), \\
\tilde{f}_{k}\left(x_{1}, x_{2}\right) & =\sum_{\left(k_{1}, k_{2}\right)} \pm \exp \left(\frac{2 i}{3}\right)\left(k_{2} x_{1}+\frac{2 k_{1}-k_{2}}{\sqrt{3}} x_{2}\right),
\end{aligned}
$$

and for $\tilde{\Omega}_{3} \subset \mathbb{R}^{3}$,

$$
\begin{aligned}
\tilde{\lambda}_{k} & =\frac{16}{27}\left(k_{1}^{2}+k_{2}^{2}-k_{1} k_{2}\right)+\frac{k_{3}^{2}}{L^{2}}, \\
\tilde{f}_{k}\left(x_{1}, x_{2}, x_{3}\right) & =\sin \frac{k_{3}}{L} x_{3} \sum_{\left(k_{1}, k_{2}\right)} \pm \exp \left(\frac{2 i}{3}\right)\left(k_{2} x_{1}+\frac{2 k_{1}-k_{2}}{\sqrt{3}} x_{2}\right),
\end{aligned}
$$

where $k=\left(k_{1}, k_{2}\right) \in \mathbb{Z}^{2}\left(k=\left(k_{1}, k_{2}, k_{3}\right) \in \mathbb{Z}^{2} \times \mathbb{Z}_{+}\right.$for $\left.n=3\right)$ satisfies (i) $K_{1}+k_{2}$ is multiple of 3 , (ii) $k_{1} \neq 2 k_{2}$, (iii) $k_{2} \neq 2 k_{1}$, and $\left(k_{1}, k_{2}\right)$ in the summation ranges over $S \subset \mathbb{Z}^{2},|S|=6$, and \pm is determined by the following rules: (for example, if $\left(K_{1}, k_{2}\right)$, $\left(k_{2}-k_{1},-k_{1}\right),\left(-k_{2}, k_{1}-k_{2}\right)$ have positive signs then the others have the negative signs).

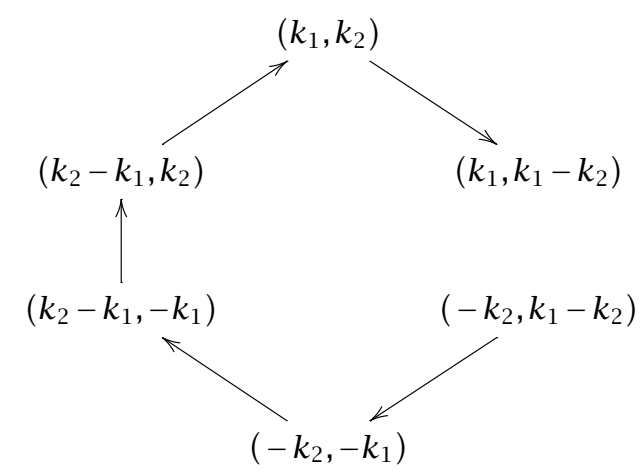

Each leg of the cycle induces a change of the sign in the $\left(k_{1}, k_{2}\right)$ entry of (2.3) and (2.4). 
For this proof, we refer to Pinsky [3] and Kwean [1].

REMARK 2.2. For each $\tilde{\Omega}_{n}, n=2,3$, if we consider the Neumann boundary conditions, then the eigenvalues $\tilde{\lambda}_{k}$ are the same as in (2.3) and (2.4) and the eigenfunction $\tilde{g}_{k}$ associated with the eigenvalue $\tilde{\lambda}_{k}$ is the same except that signs are all positive.

From the results of Lemma 2.1, one obtains the following result.

LEMMA 2.3. Let $\Omega_{n} \subset \mathbb{R}^{n}$ be given in (1.2) for $n=2,3$. Then the eigenvalues and the eigenfunctions of $-\Delta$ for given boundary conditions (1.4) are of the forms: for $\Omega_{2} \subset \mathbb{R}^{2}$,

$$
\begin{aligned}
\lambda_{k} & =\frac{16}{27}\left(k_{1}^{2}+k_{2}^{2}-k_{1} k_{2}\right), \\
f_{\left(k_{1}, k_{2}\right)}\left(x_{1}, x_{2}\right) & =\tilde{f}_{\left(k_{1}, k_{2}\right)}\left(x_{1}, x_{2}\right)+\tilde{f}_{\left(k_{2}, k_{1}\right)}\left(x_{1}, x_{2}\right),
\end{aligned}
$$

and for $\Omega_{3} \subset \mathbb{R}^{3}$,

$$
\begin{gathered}
\lambda_{k}=\frac{16}{27}\left(k_{1}^{2}+k_{2}^{2}-k_{1} k_{2}\right)+\frac{k_{3}^{2}}{L^{2}}, \\
f_{k}\left(x_{1}, x_{2}, x_{3}\right)=\sin \frac{k_{3}}{L} x_{3} f_{\left(k_{1}, k_{2}\right)}\left(x_{1}, x_{2}\right),
\end{gathered}
$$

where $k=\left(k_{1}, k_{2}\right) \in \mathbb{Z}^{2}\left(k=\left(k_{1}, k_{2}, k_{3}\right) \in \mathbb{Z}^{2} \times \mathbb{Z}_{+}\right.$for $\left.n=3\right)$ satisfies (i) $k_{1}, k_{2}$ are both multiples of 3 , (ii) $k_{1} \neq 2 k_{2}$, (iii) $k_{2} \neq 2 k_{1}$, and above $\tilde{f}_{\left(k_{1}, k_{2}\right)}$ 's in (2.6) are defined as in (2.3).

Proof. We consider a reflection operator

$$
R:\left(x_{1}, x_{2}\right) \longrightarrow\left(\pi-x_{1}, x_{2}\right) .
$$

Then for each solution $f$ of (2.1), there is an extension $\tilde{f}$ of $f$ on $\tilde{\Omega}_{n} \subset \mathbb{R}^{n}$ in (2.2) by $R \tilde{f}=\tilde{f}$ and $\left.\tilde{f}\right|_{\Omega n}=f$. Then $\tilde{f}$ is an eigenfunction of $-\Delta$ on $\tilde{\Omega}_{n}$ for Dirichlet boundary conditions. The converse is also true. However, since $R \tilde{f}_{\left(k_{1}, k_{2}\right)}=\tilde{f}_{\left(k_{2}, k_{1}\right)}$ for each $\tilde{f}_{\left(k_{1}, k_{2}\right)}$ in (2.3), the eigenfunctions of $-\Delta$ for given boundary condition (1.4) are of the form as in (2.6) and (2.7).

Now we define a multiplication operator as follows: for any $v \in L^{\infty}$, we let $B_{v}$ denote the operator on $L^{2}$ defined by

$$
\left(B_{v} u\right)(x)=v(x) u(x), \quad u \in L^{2}
$$

and let $\tilde{v}$ be the mean value

$$
\tilde{v}=(\operatorname{vol} \Omega)^{-1} \int_{\Omega} v(x) d x
$$

DEFINITION 2.4. For a given (bounded Lipschitz) domain $\Omega \subset \mathbb{R}^{n}, n \leq 3$, and choice of boundary conditions for the Laplacian, we say the weaker principle of spatial averaging (PSA) holds if there exists a quantity $\xi>0$ such that for every $\epsilon>0, \kappa<0$ and any bounded subset $\mathscr{B} \subset H^{2}$, there exists arbitrarily large $\lambda=\lambda(\mathscr{B})>\kappa$ such that

$$
\left\|\left(P_{\lambda+\kappa}-P_{\lambda-\kappa}\right)\left(B_{v}-\tilde{v} I\right)\left(P_{\lambda+\kappa}-P_{\lambda-\kappa}\right)\right\|_{\text {op }} \leq \epsilon
$$


holds for any $v \in \mathscr{B}$; such that

$$
\lambda_{m+1}-\lambda_{m} \geq \xi
$$

where $m$ satisfies $\lambda_{m} \leq \lambda<\lambda_{m+1}$.

Here we introduce two geometric properties of lattices in $\mathbb{R}^{n}, n=2,3$, which are crucial to the proof of a weaker principle of spatial averaging for each domain $\Omega_{n} \subset \mathbb{R}^{n}$ in (1.2) with (1.4).

The first property is a property related to the spectral gap proved by Mallet-Paret and Sell [2]; see also Richard [4].

LEMMA 2.5. Let $\mathcal{T}$ be a finite collection of functions $T$ of the form

$$
T\left(k_{1}, k_{2}\right)=a k_{1}^{2}+b k_{1} k_{2}+c k_{2}^{2}+s k_{1}+t k_{2}+r
$$

with rational coefficients and negative discriminant, that is, $b^{2}-4 a c<0$. Then given any $h>0$ there exists arbitrarily large $m$ such that

$$
T\left(k_{1}, k_{2}\right) \notin[m, m+h],
$$

for all $T \in \mathcal{T}$ and $k_{1}, k_{2} \in \mathbb{Z}$.

For the next theorem, we consider the three linearly independent vectors in $\mathbb{R}^{3}$ :

$$
e_{1}=\left(\frac{4}{3 \sqrt{3}}, 0,0\right), \quad e_{2}=\left(-\frac{2}{3 \sqrt{3}}, \frac{2}{3}, 0\right), \quad e_{3}=\left(0,0, \frac{1}{L}\right),
$$

and we define a new inner product and a norm induced by

$$
\langle x, y\rangle=\left(\sum_{s=1}^{3} x_{s} e_{s}, \sum_{t=1}^{3} y_{t} e_{t}\right), \quad|[x]|^{2}=\langle x, y\rangle,
$$

where $x, y \in \mathbb{R}^{3}$ and $(\cdot, \cdot)$ is usual inner product in $\mathbb{R}^{3}$.

LEMMA 2.6. Assume that $L^{2}$ is rational number. Let $k=\left(k_{1}, k_{2}, k_{3}\right) \in \mathbb{Z}^{3}$ and consider

$$
|[k]|^{2} \equiv \frac{16}{27}\left(k_{1}^{2}+k_{2}^{2}-k_{1} k_{2}\right)+\frac{k_{3}^{2}}{L^{2}}
$$

Then there exists $\xi>0$ such that for any $\kappa>1$ and $d>0$, there exists an arbitrarily large $\lambda$ satisfying two conditions:

(i) whenever $|[k]|^{2},|[l]|^{2} \in(\lambda-\kappa, \lambda+\kappa]$ with $k, l \in \mathbb{Z}^{3}$, one has either $k=l$ or $|[k-l]| \geq d$,

(ii) $|[k]|^{2} \notin(\lambda-\xi / 2, \lambda+\xi / 2)$ for each $k \in \mathbb{Z}^{3}$.

Proof. We follow Mallet-Paret and Sell's approach [2]. Let $L^{2}=q / p$, where $p$ and $q$ are relative prime integers. Let $\alpha=\operatorname{LCM}\{27, q\}$ be fixed where LCM means least common multiple. Then for any $k \in \mathbb{Z}^{3}$, there exist integers $n$ and $r$ such that

$$
|[k]|^{2}=n+\frac{r}{\alpha}, \quad 0 \leq r<\alpha
$$


Therefore, with $\xi=1 /(2 \alpha)$ we see that there are arbitrarily large $\lambda$ such that $|[k]|^{2} \notin$ $(\lambda-\xi / 2, \lambda+\xi / 2)$. For the rest of the proof we will consider only such $\lambda$. Let $\lambda$ be fixed and let $N_{0}^{\lambda}$ be the annular region

$$
N_{0}^{\lambda} \equiv\left\{x \in \mathbb{R}^{3}: \lambda-\kappa<|[x]|^{2} \leq \lambda+\kappa\right\} .
$$

Suppose that $k, l \in N_{0}^{\lambda} \cap \mathbb{Z}^{3}$ and $0 \leq|[k-l]|<d$. Then for $j=l-k$,

$$
|[l]|^{2}=|[j+k]|^{2}=|[j]|^{2}+2\langle k, j\rangle+|[k]|^{2},
$$

where $\langle\cdot, \cdot\rangle$ is defined in (2.16). As a result,

$$
\begin{aligned}
|\langle k, j\rangle| & \leq\left.\frac{1}{2}||[l]\right|^{2}-|[k]|^{2}-|[j]|^{2} \mid \\
& \leq\left.\frac{1}{2}||[l]\right|^{2}-\left.|[k]|^{2}\left|+\frac{1}{2}\right|[j]\right|^{2} \\
& <\kappa+\frac{d^{2}}{2} .
\end{aligned}
$$

For each $j$ with $0<|[j]|<d$, let $S_{j}=\left\{x \in \mathbb{R}^{3}:|\langle x, j\rangle|<\kappa+d^{2} / 2\right\}$ and let $S=$ $\bigcup_{0<|[j]|<d} S_{j}$. If the property (i) fails for some $\lambda$, then $S \cap N_{0}^{\lambda} \cap \mathbb{Z}^{3} \neq \varnothing$. If $k \in S \cap N_{0}^{\lambda} \cap \mathbb{Z}^{3}$, then

$$
\begin{gathered}
|\langle k, j\rangle|<\kappa+\frac{d^{2}}{2}, \\
\gamma \equiv\langle k, j\rangle=\frac{8 k_{1}}{27}\left(2 j_{1}-j_{2}\right)+\frac{8 k_{2}}{27}\left(2 j_{2}-j_{1}\right)+\frac{k_{3} j_{3}}{L^{2}},
\end{gathered}
$$

for some $j$ and some $\gamma=n / \alpha$, where $0<|[j]|<d$ and $n$ is an integer such that $|n / \alpha|<\kappa+d^{2} / 2$. Since $\gamma=n / \alpha$ for some integer $n$, there is only a finite number of $\gamma$ satisfying $|\gamma|=|n / \alpha|<\kappa+d^{2} / 2$. On the other hand since $j \neq 0$, we may assume that $j_{3} \neq 0$. Then by solving $\langle k, j\rangle=\gamma$ for $k_{3}$, it is found that

$$
k_{3}=-\frac{L^{2}}{j_{3}}\left(\frac{8 k_{1}}{27}\left(2 j_{1}-j_{2}\right)+\frac{8 k_{2}}{27}\left(2 j_{2}-j_{1}\right)-\gamma\right)
$$

and hence by substituting $k_{3}$,

$$
\begin{aligned}
|[k]|^{2}= & \frac{16}{27}\left(k_{1}^{2}+k_{2}^{2}-k_{1} k_{2}\right)+\frac{k_{3}^{2}}{L^{2}} \\
= & \left(\frac{16}{27}+\frac{L^{2}}{j_{3}^{2}}\left(\left(\frac{16}{27}\right)^{2} j_{1}^{2}+\left(\frac{16}{27}\right)^{2} j_{2}^{2}-\left(\frac{16}{27}\right)^{2} j_{1} j_{2}\right)\right) k_{1}^{2} \\
& +\left(-\frac{16}{27}+\frac{L^{2}}{j_{3}^{2}}\left(2\left(\frac{8}{27}\right)^{2} j_{1} j_{2}-\left(\frac{16}{27}\right)^{2} j_{1}^{2}+2\left(\frac{16}{27}\right)^{2} j_{1} j_{2}-\left(\frac{8}{27}\right)^{2} j_{2}^{2}\right)\right) k_{1} k_{2} \\
& +\left(\frac{16}{27}+\frac{L^{2}}{j_{3}^{2}}\left(\left(\frac{8}{27}\right)^{2} j_{1}^{2}+\left(\frac{16}{27}\right)^{2} j_{2}^{2}-\left(\frac{16}{27}\right)^{2} j_{1} j_{2}\right)\right) k_{2}^{2} \\
& +s_{j, \gamma} k_{1}+t_{j, \gamma} k_{2}+r_{j, \gamma},
\end{aligned}
$$


where $s_{j, \gamma}, t_{j, \gamma}$, and $r_{j, \gamma}$ are rationals depending only on $j$ and $\gamma$. Now by taking coefficients in (2.24), we define a quadratic function $T_{j, \gamma}$ on $\mathbb{Z}^{2}$ with rational coefficients of the form

$$
T_{j, \gamma}\left(l_{1}, l_{2}\right)=a_{j} l_{1}^{2}+b_{j} l_{1} l_{2}+c_{j} l_{2}^{2}+s_{j, \gamma} l_{1}+t_{j, \gamma} l_{2}+r_{j, \gamma} .
$$

Then the discriminant of $T_{j, y}$ in (2.25) is negative. Also since $k \in N_{0}^{\lambda}$,

$$
T_{j, \gamma}\left(k_{1}, k_{2}\right) \in(\lambda-\kappa, \lambda+\kappa] .
$$

Now let $\mathcal{T}$ be the set of all quadratic functions $T_{j, \gamma}$ of the form in (2.25) for $j \in \mathbb{Z}^{3}$ and $\gamma=n / \alpha$ with $0<|[j]|<d,|\gamma|=|n / \alpha|<\kappa+d^{2} / 2$. Then since the indices $j$ and $\gamma$ range over finite sets, $\mathcal{T}$ is a finite collection of functions $T_{j, \gamma}$ satisfying all hypotheses of Lemma 2.5. With $h=2+2 \kappa$, there exists a $m$ in the statement of Lemma 2.5 such that for any $T_{j, \gamma} \in \mathscr{T}$ and $l \in \mathbb{Z}^{2}$

$$
T_{j, \gamma}\left(l_{1}, l_{2}\right) \notin[m, m+h], \quad(\lambda-\kappa, \lambda+\kappa) \subset[m, m+h],
$$

for some $\lambda$ satisfying the second assertion (ii). Therefore (2.26) is impossible for this $\lambda$. As $m$ can be chosen arbitrarily large, the proof is now complete.

From these lemmas, we obtain the following important results.

LEMMA 2.7. Let $\Omega_{3}$ be given in (1.2). Fix the boundary conditions (1.4) for $n=3$. Let $\Re$ be a bounded subset of $H^{2}$. Then for any $\epsilon>0$ and $\kappa>1$, there exists arbitrarily large $\lambda=\lambda(\mathscr{B})>\kappa$ such that

$$
\left|\int_{\Omega 3}(v-\tilde{v}) \rho^{2} d x\right| \leq \epsilon
$$

for any $v \in \mathscr{B}$ and $\rho \in \operatorname{Range}\left(P_{\lambda+\kappa}-P_{\lambda-\kappa}\right) \subset L^{2}$ with $\|\rho\|=1$.

Proof. We note that the product of any two eigenfunctions of the form in (2.7) is a finite combinations of eigenfunctions $\tilde{g}_{k-l}$ of $-\Delta$ for Neumann boundary conditions as we mention in Remark 2.2, that is,

$$
f_{k} \bar{f}_{l}=\tilde{g}_{k-l},
$$

where $\bar{f}$ means the complex conjugate of $f$. With the property (2.29), the result follows from property (1) of Lemma 2.6 and the facts that the set of eigenfunctions of Laplace operator forms complete orthogonal basis for $L^{2}$ and that any bounded set of $H^{2}$ is a compact subset of $L^{2}$ for $n \leq 3$. For more detail proof, we mention Kwean [1].

By combining the results of the previous lemmas, one obtains the following theorem.

THEOREM 2.8. The weaker PSA holds for the domains $\Omega_{n}, n=2,3$ in (1.2) with given boundary conditions (1.4).

Proof. We fix a quantity $\xi>0$ satisfying property (2) of Lemma 2.6. Let $\epsilon>0$, $\kappa>0$, and a bounded subset $\mathscr{B} \subset H^{2}\left(\Omega_{n}\right)$ be given. Then we have arbitrarily large $\lambda>\kappa$ satisfying property (1) of Lemma 2.5 and inequalities (2.28) in Lemma 2.7. Therefore inequalities (2.11) and (2.12) can be obtained by the choices of $\xi>0$ and $\lambda$. 
3. Inertial manifold. We are ready to examine the existence of inertial manifold of the equation of the form

$$
\frac{\partial u}{\partial t}=v \Delta u+f(u), \quad u \in \mathbb{R}
$$

where the domains $\Omega_{n} \subset \mathbb{R}^{n}$ are given in (1.2). For $n=3$, we assume that $L^{2}$ is rational. Moreover, the nonlinearity

$$
f: \mathbb{R} \rightarrow \mathbb{R}
$$

is assumed to satisfy the following conditions for some positive constants $K_{1}$ and $K_{2}$;

$$
\begin{gathered}
f \text { is } C^{1} \quad \text { in } \mathbb{R}, \\
|f(u)| \leq K_{1}|u|+K_{2} \quad \text { in } \mathbb{R}, \\
\left|D_{u} f(u)\right| \leq K_{1} \quad \text { in } \mathbb{R},
\end{gathered}
$$

and we consider the boundary conditions for (3.1); for each $n=2,3$,

$$
u=0 \quad \text { on } S_{n}^{c}, \quad \frac{\partial u}{\partial n}=0 \quad \text { on } S_{n} .
$$

Then we can assert the following.

THEOREM 3.1. Assume that (3.1) is dissipative and that $f: \mathbb{R} \rightarrow \mathbb{R}$ is of class $C^{3}$ satisfying conditions (3.3). Fix the boundary conditions (3.4). Then for every $v>0$ there exists an inertial manifold $M$ for (3.1).

Proof. Due to the invariant manifold theorem [2] and the dissipativity of (3.1), we can prove the existence of an inertial manifold $\mathcal{M}$ (see [1]).

ACKNOWLEDGEMENT. This work was supported by the Korea university grant.

\section{REFERENCES}

[1] H. Kwean, An extension of the principle of spatial averaging for inertial manifolds, J. Austral. Math. Soc. Ser. A 66 (1999), no. 1, 125-142. MR 2000a:35124. Zbl 0952.35011.

[2] J. Mallet-Paret and G. R. Sell, Inertial manifolds for reaction diffusion equations in higher space dimensions, J. Amer. Math. Soc. 1 (1988), no. 4, 804-866. MR 90h:58056. Zbl 0674.35049.

[3] M. A. Pinsky, The eigenvalues of an equilateral triangle, SIAM J. Math. Anal. 11 (1980), no. 5, 819-827. MR 82d:35077. Zbl 0462.35072.

[4] I. Richards, On the gaps between numbers which are sums of two squares, Adv. in Math. 46 (1982), no. 1, 1-2. MR 84d:10055. Zbl 0501.10047.

HyukJin KWean: Department of Mathematics Education, College of Education, Korea University, 1, 5-KA, ANAm-Dong, Sungbuk-Ku, 136-701, Seoul, Korea

E-mail address: kwean@mai 1.korea.ac.kr 


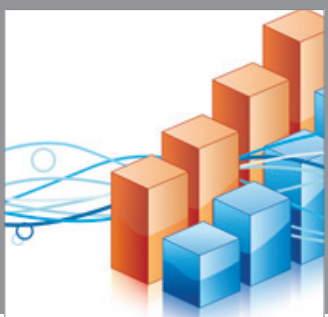

Advances in

Operations Research

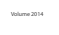

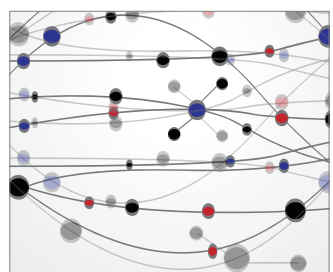

\section{The Scientific} World Journal
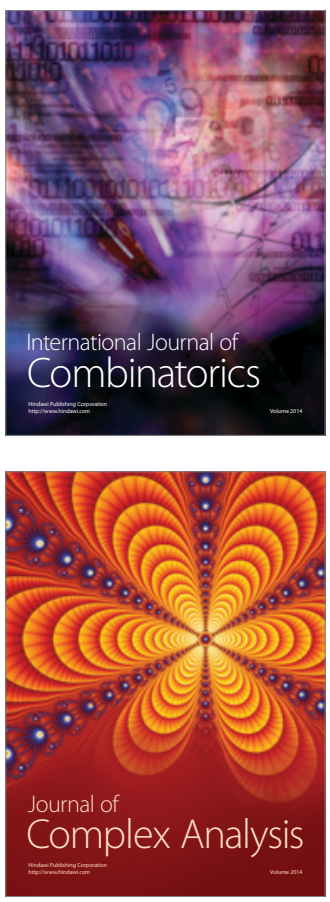

International Journal of

Mathematics and

Mathematical

Sciences
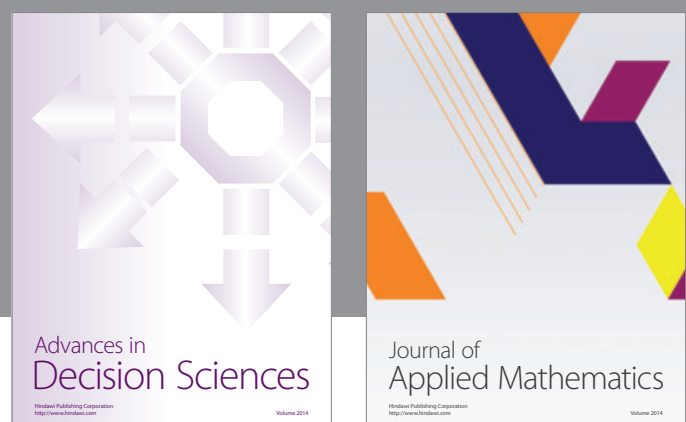

Journal of

Applied Mathematics
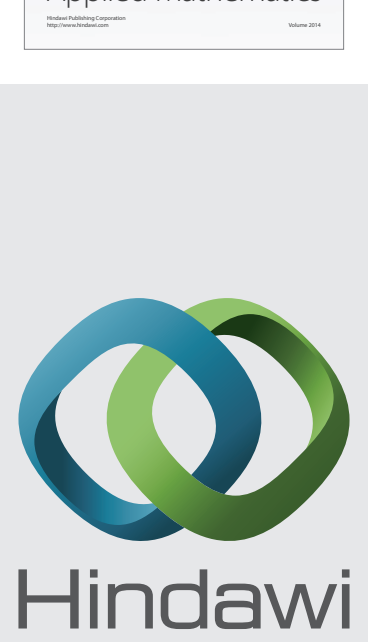

Submit your manuscripts at http://www.hindawi.com
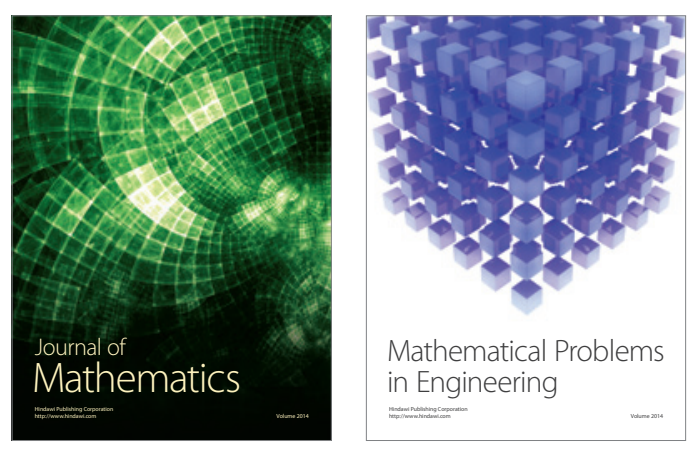

Mathematical Problems in Engineering
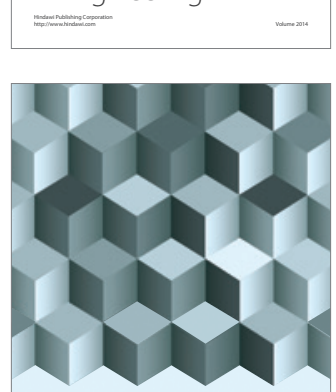

Journal of

Function Spaces
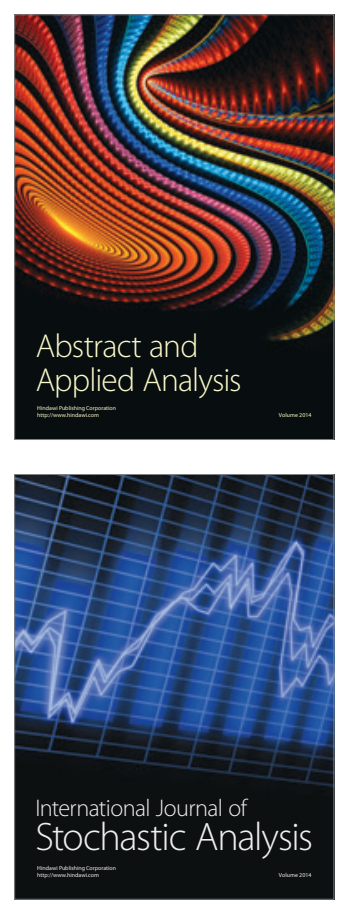

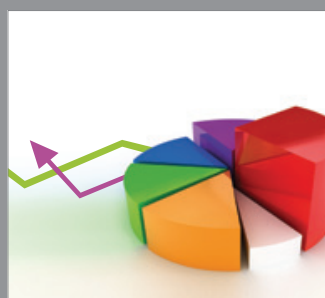

ournal of

Probability and Statistics

Promensencen
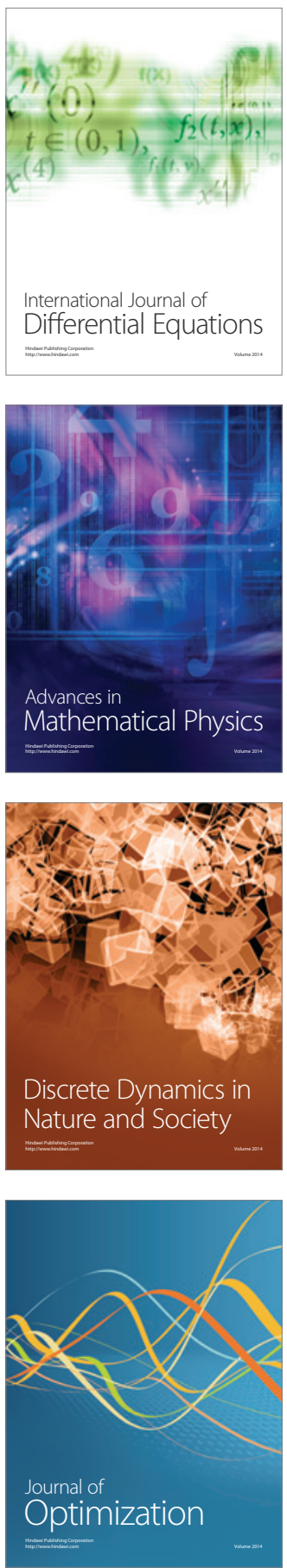